\title{
The Status of Antibiotics Pollution in Coastal Wetlands in China
}

\author{
Ming-yue Zhang a , Chun-qiu Ran ${ }^{b, *}$, Ze-hang Huang ${ }^{c}$, Shi-fei Lid $^{\mathrm{d}}$ Yi-man Niu${ }^{\mathrm{e}}$, \\ Yu-bo Cuib
}

\author{
College of Environment and Resources, Dalian Minzu University, Dalian, China, \\ ${ }^{*}$ Corresponding author \\ a939384425@qq.com, brancq@dlnu.edu.cn, chuangzehang1123@hotmail.com,

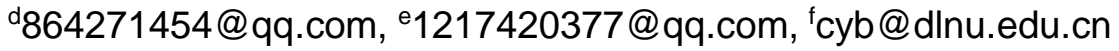

\begin{abstract}
Keywords: antibiotic; residual; ecological risk; reduction measures
Abstract. In recent years, drugs have been classified as new pollutants, and an increasing attention has been paid in the field of environmental remediation. As an effective drug for treating diseases and promoting growth, antibiotics are widely used in medical, livestock and aquaculture. However, due to the improper use and excessive abuse, in addition to directly pollution to the environment caused by themselves, antibiotics also lead to the transmission of antibiotic resistant bacteria and antibiotic resistance gene, which threat aquatic growing in the natural environment and human health. Recent studies have found that antibiotics are retained in soil, wastewater and sludge, and the residual species are complex. Therefore, it is essential to explore the origin and the fate of antibiotics in the marine environment. The paper mainly introduced the current research status of antibiotics in coastal wetlands in China, and analyzed the potential ecological risks of antibiotics to marine and aquaculture environment so as to put forward countermeasures to reduce them.
\end{abstract}

\section{Introduction}

The initial definition of antibiotics refers to those substance produced by microorganisms, had relative selective toxicity, and could restrain the growth and reproduction of pathogenic microorganisms (or cancer cells) at certain concentrations. Nowadays, the variety of antibiotics is more complex, mainly including anti-bacterial antibiotics, anti-fungal antibiotics, antiviral antibiotics, antitumor antibiotics and so on. In recent years, the use of antibiotics has increased dramatically in the world, and the annual usage of antibiotics in China accounts for $1 / 4$ of the total in the world. The number of antibiotics can be not underestimated. Antibiotics can enhance the induction of bacterial resistance to bacteria and then potentially threaten public health. Antibiotics continue to enter the water body through different ways, causing the "false persistence" of antibiotics in the water environment. The residues of antibiotics in marine environment are various and complex. They will have different effects on the marine water environment, microbial communities, plants and aquatic animals. Therefore, it is necessary to explore the sources and trends of antibiotics in the marine environment, and to assess their potential environmental and health risks.

\section{The Source of Antibiotics in Marine Environment}

The marine environment is the main region of antibiotic residues. The source of pollution mainly includes internal source and point source. Although the process in water, such as dilution, adsorption, hydrolysis, photolysis and biodegradation can reduce the concentration of antibiotic residues, it can be not completely removed. 
Endogenous Pollution. Marine microbes include marine bacteria, marine fungi, and marine actinomycetes, whose types are about 20 times more than terrestrial microorganisms. Because of their special living environment (oligosaccharides, low light, low temperature and high pressure), marine microbes can synthesize some new kind antibiotic, which is not available by terrestrial microbes[1].It is known that many marine microorganisms can produce antibiotics, including actinomyces, air monobacillus, micrococcus, actinomyces and many undetermined bacteria. The produced antibiotics include peptide, alkaloids, lipids, glycosides and other structural types.

Point Source Pollution. The sewage treatment plant, as a point source pollution, has a great influence on the emission and distribution of antibiotics. At present, quinolones are the main pollutants in sewage treatment plants, which also indicate that quinolones are difficult to degrade. But the process of sewage treatment plant, such as activated sludge process [2], has some effect on the removal of some antibiotics, but the removal efficiency of quinolone antibiotics is low.

The pollution of aquatic antibiotics in aquaculture areas is particularly obvious. In the detection of antibiotics pollution in aquaculture waters in Southern China Sea and the Yellow Sea, the antibiotic residues are found in all detected bodies. Quinolones and tetracycline are the main antibiotic pollutants in aquaculture areas. In addition, with the increase of salinity, the concentration of dissolved organic carbon in water decreased. As a substance with strong adsorptive capacity, the reduction of dissolved organic carbon in water may lead to a small amount of antibiotic adsorption and increase the number of dissolved antibiotics in water. The point source pollution caused by the intensity of human activity is also the main cause of the distribution of antibiotics in the environment. The research of Li Jia and other people on the antibiotics distribution of the Xiaoqing River basin surface water shows that sewage can usually be detected with high concentration of antibiotic [3], and the sewage mainly from the residential area, especially in densely populated areas, so densely populated area is often the hardest hit people with antibiotics.

\section{Pollution Status of Antibiotics in Marine Environment}

Antibiotics are widespread in the marine environment. In modern aquaculture industry, a substantial number of antibiotics are used for the prevention and treatment of fish diseases and to accelerate the growth of fish. Those being released into the water without eaten and then excreted into the water will eventually become pollution. It is estimated that at least $75 \%$ of the antibiotics used in aquaculture will enter the water body and form accumulative pollution in the sediment. These antibiotics mainly include sulfonamides, quinolones, tetracycline and so on.

From the current researches, the most common types of antibiotics in water are sulfonamides, quinolones, and macrolides. Tetracycline antibiotics are also often detected. The different pollution degree may because(1) Different use amount of antibiotics in water environment;(2) Different antibiotics have different metabolicrate in animal and human body; (3) The characteristics of transport, transformation and migration in the environment are different; (4) The enrichment of samples in the present study has different recovery rates.

\section{Biodegradation Pathway of Antibiotics in Marine Environment}

Antibiotics will undergo a series of degradation reactions in the environment. The degradation of antibiotics can be divided into biological and abiotic processes [4], non-biodegradation, including hydrolysis, oxidative degradation, photo degradation and so on. Biodegradation processes mainly include plant degradation and microbial degradation. In the process of degradation, antibiotics are converted from macromolecular compounds to small molecular compounds and eventually 
converted into water and carbon dioxide. However, antibiotics that enter the environment are hard to be completely degraded, instead they produce a series of metabolites and degradation products, which often have more toxic and even cause more serious secondary pollution than the parent compounds. As a result, all the countries have explored the best ways to degrade antibiotics and try to minimize the pollution of antibiotics.

\section{Ecological Risks and Health Risks Caused by Antibiotic Residues in Marine Environment}

In addition to directly causing chemical pollution in the environment, antibiotics will also lead to the transmission of antibiotic resistant bacteria and antibiotic resistance gene (ARGs)[5].Because antibiotics provide a persistent resistance selection pressure and maintenance force for bacteria. The drug resistance of bacteria is increased and the antibiotic resistance of antibiotics is reduced or completely failed, and then the safety of human health and environmental ecology is seriously threatened.

At present, the ecological risk assessment of antibiotics is mainly concentrated on water bodies. Whether it is degraded in water, which threatens aquatic organisms, or through food chain, antibiotic residues in water will bring huge potential risks. Sulfadiazine, trimethoprim, oxytetracycline, tetracycline and other seven antibiotics have at least a low ecological risk for sensitive aquatic organisms in seawater and aquaculture water. Among them, methoxy pyrimidine and tetracyclineare highly risk factors for aquatic organisms in seawater.

Marine water body is the main carrier of antibiotic residues. In the past, most of the researches focused on the existence of antibiotics in the marine environment. In recent years, the understanding of the antibiotic pollution in aquaculture areas and its surrounding areas has been gradually deepened by scholars from various countries.

Aquaculture and antibiotic containing wastewater may be discharged into external waters, causing potential ecological risks to aquatic environment including aquatic organisms. Chen Hui and other people studied the bioaccumulation of antibiotics and human dietary nutrition in typical mariculture around Hailing Island, Southern China. The research showed that the main antibiotics in shrimp seedlings pond could not be detected in the adult shrimp pond water. The reason can be interpreted as the examination of antibiotic residues in adult shrimp was more rigorous [6].Marine products with antibiotic residues accumulate in human body in the form of food chain. Although the residual concentration of antibiotics in marine products is very low at present, the consumption quantity and frequency of food will have a subtle influence on human health. Therefore, people should pay attention to it.

The antibiotic resistance in the water body makes the microbes and bacteria drug tolerance, which makes the effect of the drug treatment reduce or lost, and increases the difficulty of patient's treatment and medical cost. After the absorption of antibiotics into the food chain through plants, the influence will further spread, which will eventually reduce the body's immunity and form a drug resistant body.

\section{Conclusion}

Marine antibiotic pollution is only a small part of a much larger problem of antibiotic pollution. Therefore, we must pay attention to the potential ecological risks caused by antibiotics. European Union countries have now completely banned the addition of any antibioticin the feed [7], so China should also put the corresponding regulations to strictly restrict the use of antibiotics in animal and 
aquaculture in time. In addition, we should increase the research on antibiotic substitutes in the field of aquaculture.

In this field of research, there is still a lot of scope for development and many problems have to be solved. In the future, some suggestions has made to do a good job in the following aspects:(1) to increase the research on low cost and less time-consuming detection methods in order to carry out routine monitoring of antibiotic pollution in a wide range; (2) to strengthen the research on the metabolism of antibiotics in the body and its transport and transformation in the environment; (3) to actively explore the possible environmental effects and biological effects of antibiotics pollution;(4) to actively develop sewage treatment technology that has high scavenging efficiency for all kinds of active compounds in sewage, including antibiotics.

\section{Acknowledgements}

The research was financed by the National Natural Science Foundation of China and the Fundamental Research Funds for the Central Universities in China.

\section{Reference}

[1] B.H. Jiao, J. Mu, Q.Z. Xu: Journal of Agro-Environment Science. Vol. 54 (2014), p. $4302-4419$

[2] H.Y. Jiang, M.D. Zhang, R.J. Zhou, Y. Yang: Water Research. Vol. 32(2012), p. 103-107

[3] J. Li, R.J. Zhang: Agro-Environment Science. Vol. 23 (2014), p. 55-58

[4] Q.F. Liu, H.L. Hong: Safely and Environmental Engineering. Vol. 17(2010), p. 68-72

[5] F. Liu, R. Tao, G.G. Ying: Agro-Environment Science. Vol. 30(2010), p. 4503-4519

[6] J. Li, H. Chen, L. Shan: Marine Pollution Bulletin. Vol. 90(2015), p. 181-187

[7] W.P. Chen, C.W. Peng, Y. Yang, Y.M. Wu: Environmental Science. Vol. 12(2017), p. 55-62 